\title{
NKIT
}

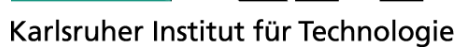

\section{Accelerating COBAYA3 on multi-core CPU and GPU systems using PARALUTION}

\section{N. Trost, J. Jimenez, D. Lukarski, V. Sanchez}

Institute for Neutron Physics and Reactor Technology (INR)

Reactor Physics and Dynamic Group (RPD)

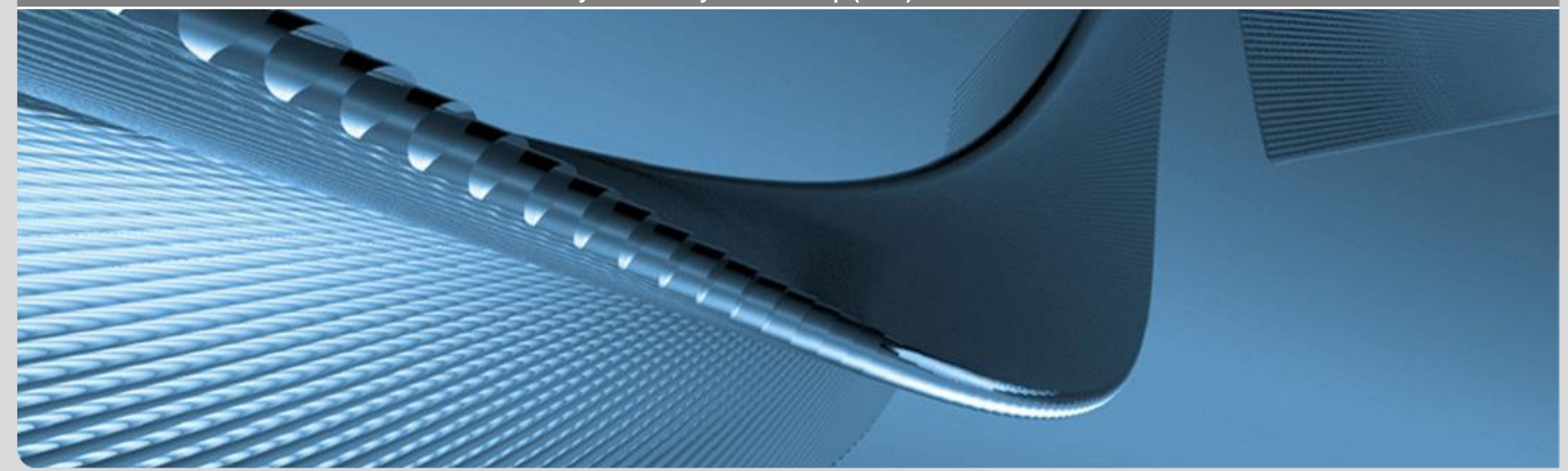




\section{Content}

- Motivation

- COBAYA3 ANDES solver

- PARALUTION

- Implementation

- Performance results

- Conclusion 


\section{Motivation}

- Multi/many-core systems are here

- CPUs, GPUs, Accelerators

- Trend: more cores CPU(16), MIC(60), GPU(2496)

- High performance, better performance/watt ratio

- Scientific computing is expected to be

- Fast - bleeding edge technology

- Scalable - exploit the available hardware resources

- Sustainable - over years without the necessity of development work

- But: How to adapt well established simulation tools, such as COBAYA3? 


\section{COBAYA3 ANDES solver (UPM)}

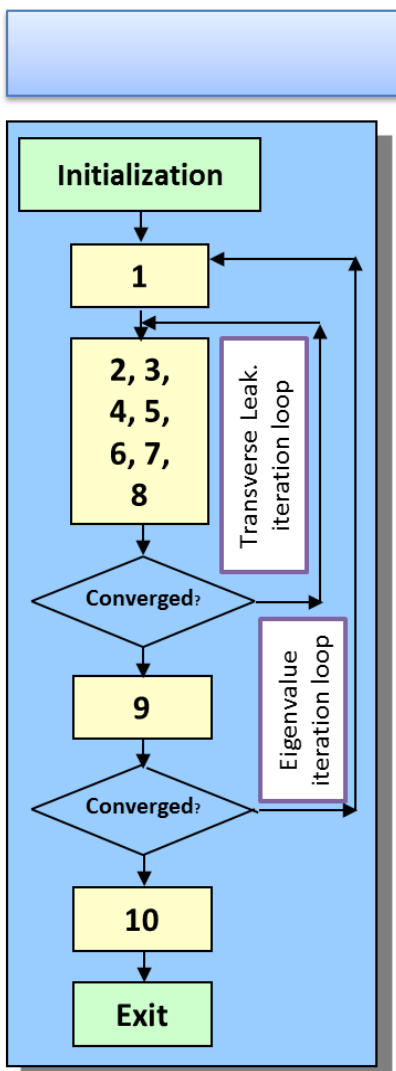

\section{ANDES CODE STRUCTURE}

0 . Input data and initialization

1. Multigroup eigenvalues and analytic matrices

2. Interpolation of group interface currents at arista's

3. Directional transverse leakage and ACMFD NGxNG matrices per interface and node

4. Heterogeneity factors (if local solution and first pass)

5. Setup of the linear system matrix in nodal fluxes

6. Build and apply pre-conditioner $\longleftarrow$ GQRARAUTION

7. Resolution of the resulting linear system

8. Update of nodal interface currents

Test for convergence of transverse leakage

9. Update of fission source [Keff] and nodal powers

Test convergence (if steady-state eigenvalue)

10. Interface average fluxes (for local reconstruction)

Exit from nodal solver

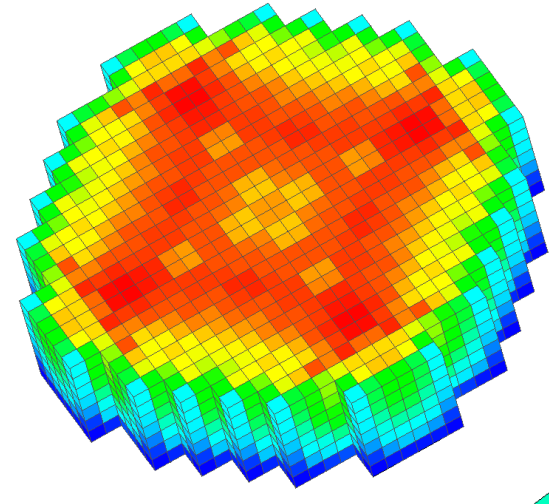

NODAL 3D

Power distribution

- Nodal solver for 3D neutron multigroup diffusion (ACMFD method)

- Available sequential solver:

- GMRES preconditioned with incomplete LU factorization based on threshold with drop off tolerance for additional fill ins 
- C++ library for iterative sparse methods

- Developed at Uppsala University (Sweden)

- Hardware abstraction, no knowledge in CUDA, OpenCL, etc. required

- Support for many/multi-core CPU and GPU devices

- High portability by design, same code can be compiled on CPU and GPU architectures

- Binary can be executed on any platform, obtaining same results

- Hardware abstraction, no knowledge in CUDA, OpenCL, etc. required

- Variety of different solvers and preconditioners

- Many different matrix formats

- CSR, MCSR, HYB, ELL, DIA, COO 


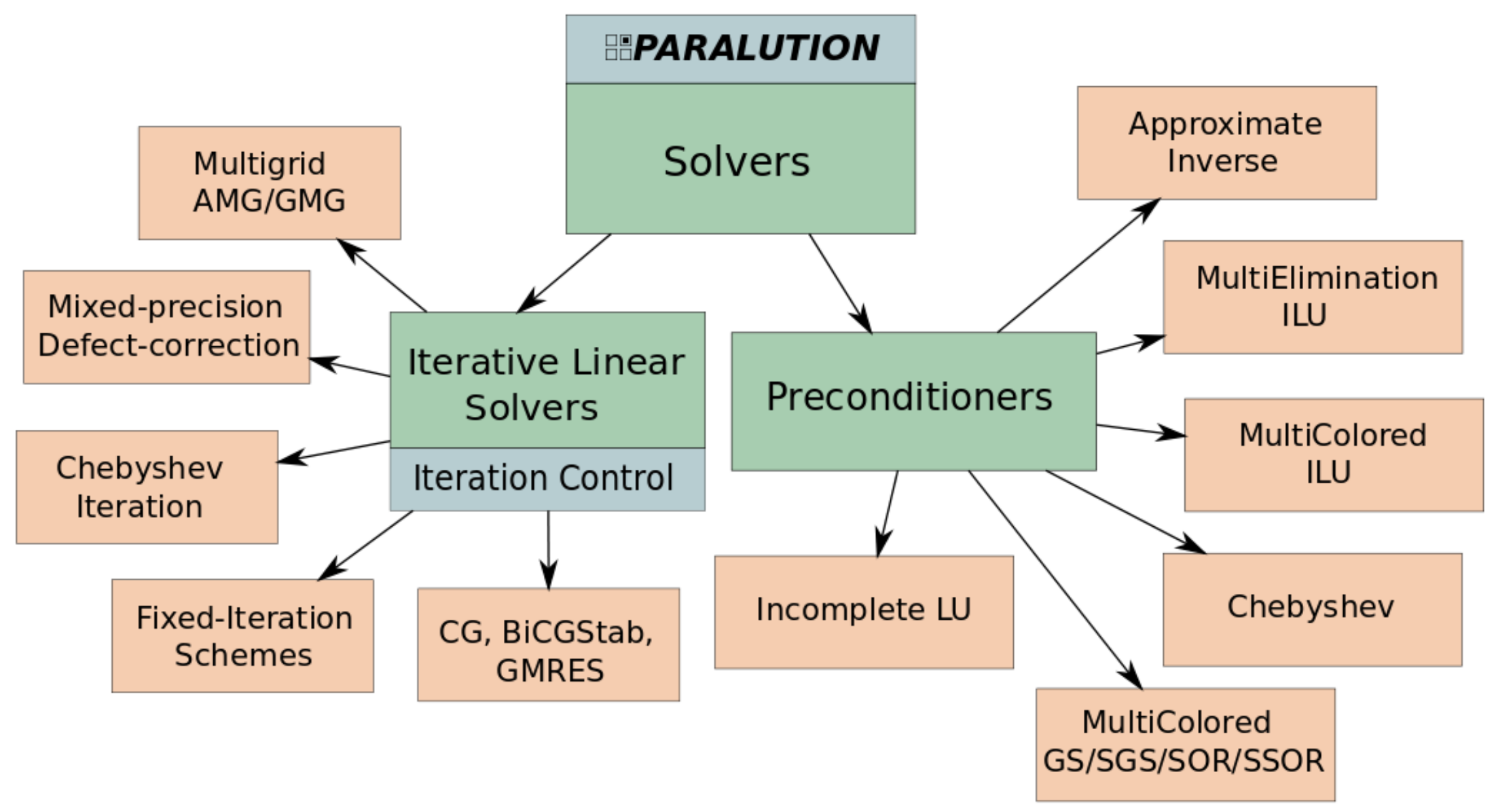




\section{Implementation aspects}

- Extension of ANDES

- Implementation of PARALUTION as an external library with support for all available solvers, preconditioners and matrix formats

- Investigation of communication between FORTRAN and $\mathrm{C}++$ with respect to

- communication overhead; index shift, separate address space (CUDA, OpenCL)

- object passing

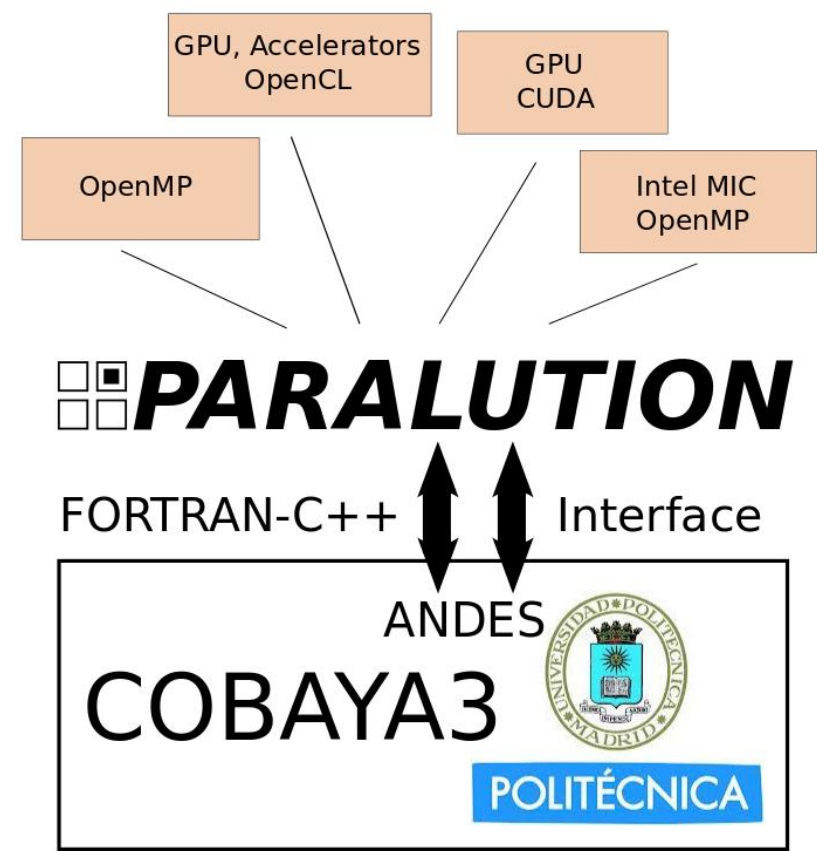




\section{Interaction FORTRAN $\leftarrow \rightarrow \mathbf{C + +}$}

- ISO_C_BINDING intrinsic allows

- C types in FORTRAN: C_INT, C_DOUBLE, C_PTR, C_CHAR

- Passing of targetable FORTRAN data structures: C_LOC, C_FUNLOC

- Passing of ANY C++ data structures / objects

- Minimization of data transfers by passing pointers

- Index shift still necessary

- Possibility to 'keep' allocated C++ objects in FORTRAN beyond C++ function execution time for e.g. re-use of preconditioners

- This also applies for device pointers in foreign memory regions (CUDA) 


\section{GPU FORTRAN $\leftrightarrow$ PARALUTION interface}

- Issues:

- Separate address space explicit data transfer necessary

- Bottleneck PCI-E bus

- Solution:

- Asynchronous data transfer (CC $>=2.0$ support asynchronous kernel call and data transfer) while shifting FORTRAN arrays to 0 -based indexing

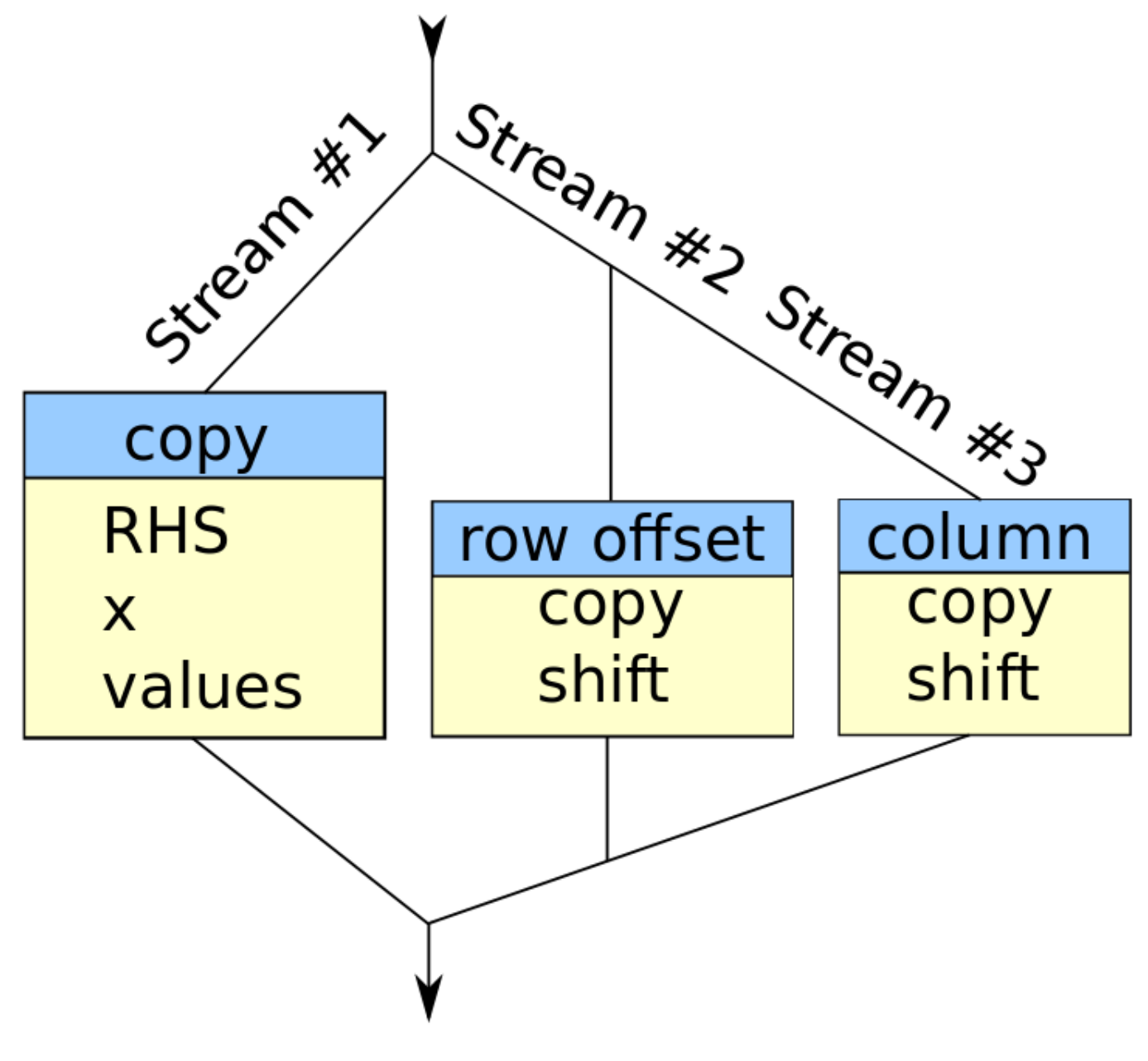




\section{Reusage of the preconditioner system - to further accelerate the solving process}

- Constant mesh and neighboring relations $\rightarrow$ systems sparsity pattern will not change

$\rightarrow$ preconditioning structure can be kept

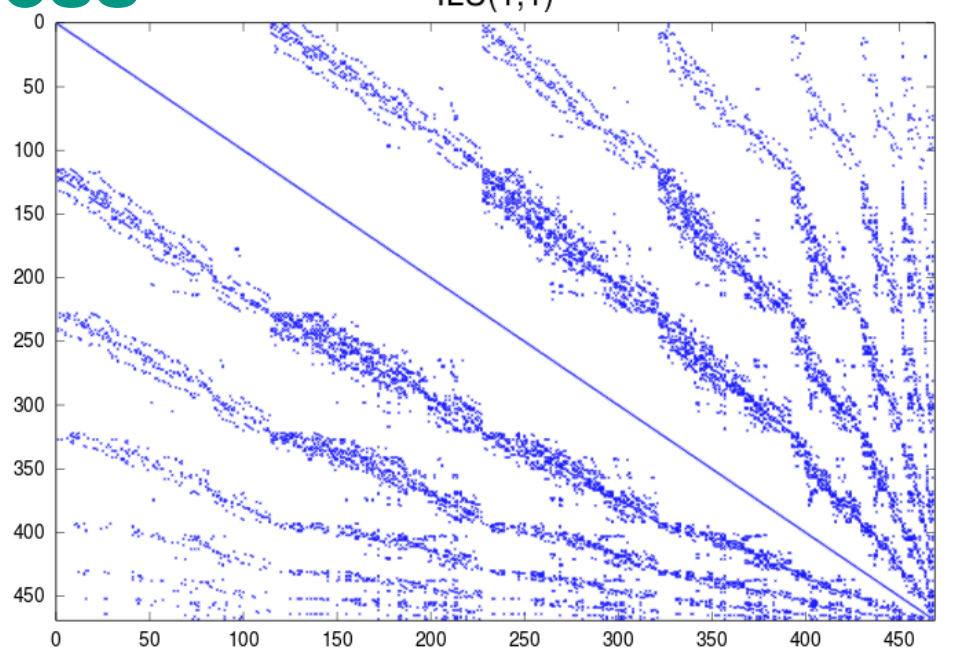

- Only minor changes in the system matrix each solver call

$\rightarrow$ preconditioning operator can be applied for a certain solver call interval

$\rightarrow$ Not only the solving process but also the building of the solver structure can be accelerated.

- Implementation:

- Fixed preconditioner rebuild interval and

- Error fall back logic (max iter reach, diverged, ... ) 


\section{Performance results}

- Test case - MOX-3D-HFP.D:

- 8 energy groups

- $4 \times 4$ nodes per assembly (Cartesian geometry)

- 20 axial layers

- Matrix dimensions: $\mathrm{n}=\mathrm{m}=723,712$

- $\mathrm{nnz}=39,618,560$

- Application of GMRES solver

- with multi-colored ILU-0 - a priori known sparsity pattern (PARALUTION)

- with ILU-T (SPARSKIT, University of Minnesota)

- ELL format (GPU), CSR format (CPU) for both, system and preconditioning operator 


\section{Performance results}

- Test system:

- Dual-socket Intel Xeon E5-2680, 2.7GHz (Sandy Bridge EP), 16 physical cores (8 per socket), approx. $3200 €$ (CPUs only)

- 64GB ECC DDR3-1600 (quad), 51.2GB/s (peak)

- GPUs:

- NVIDIA Tesla K20 (Kepler), 5GB ECC GDDR5, approx. $3200 €$

- NVIDIA GTX560Ti, 2GB GDDR5, approx. 200€ (released in 2010)

- Backends:

- PARALUTION CPU: OpenMP using up to 16 threads

- PARALUTION GPU: CUDA

- SPARSKIT: sequential using 1 core 


\section{Performance results - MOX-3D-HFP.D}

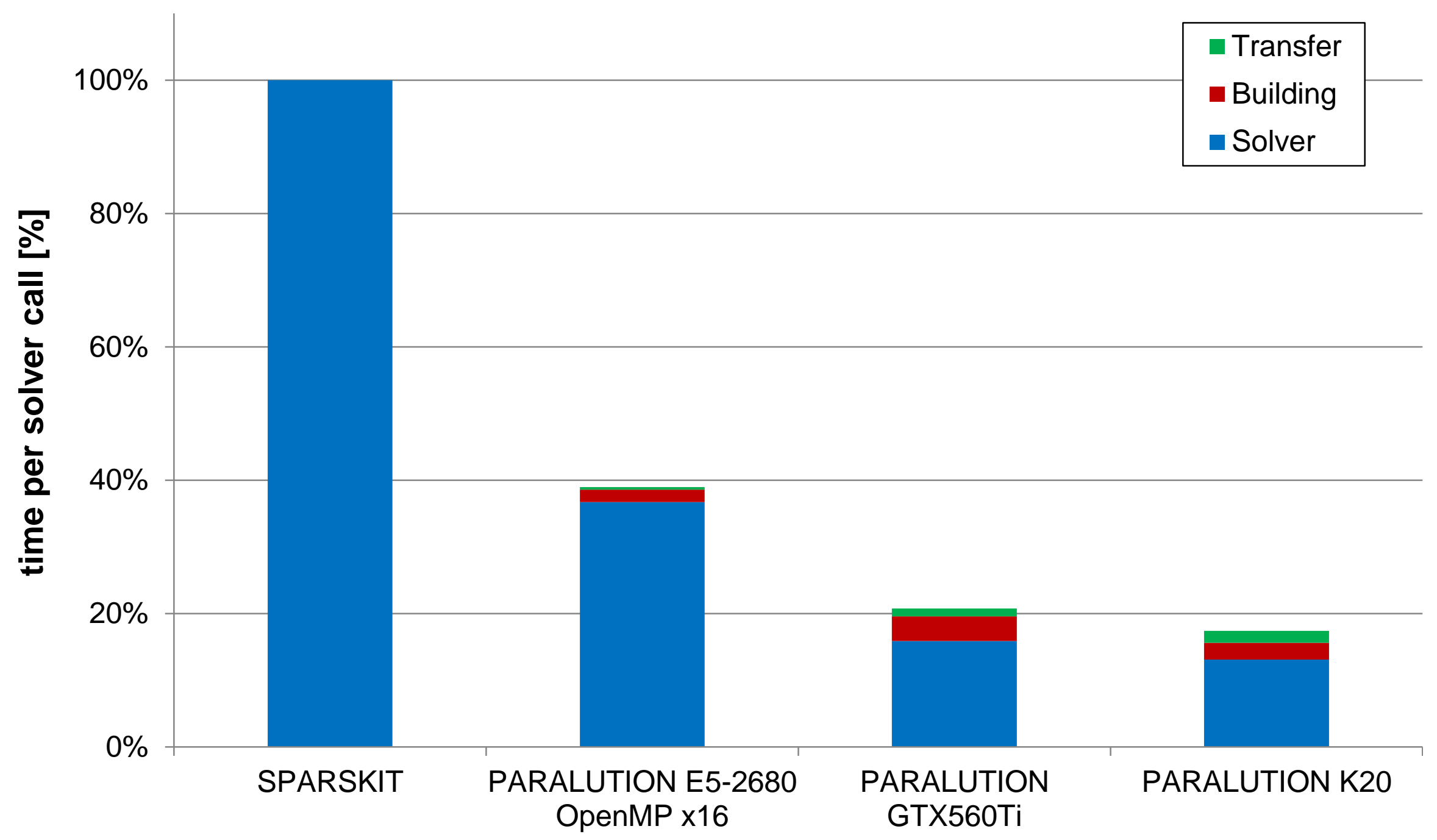




\section{Performance results - MOX-3D-HFP.D}

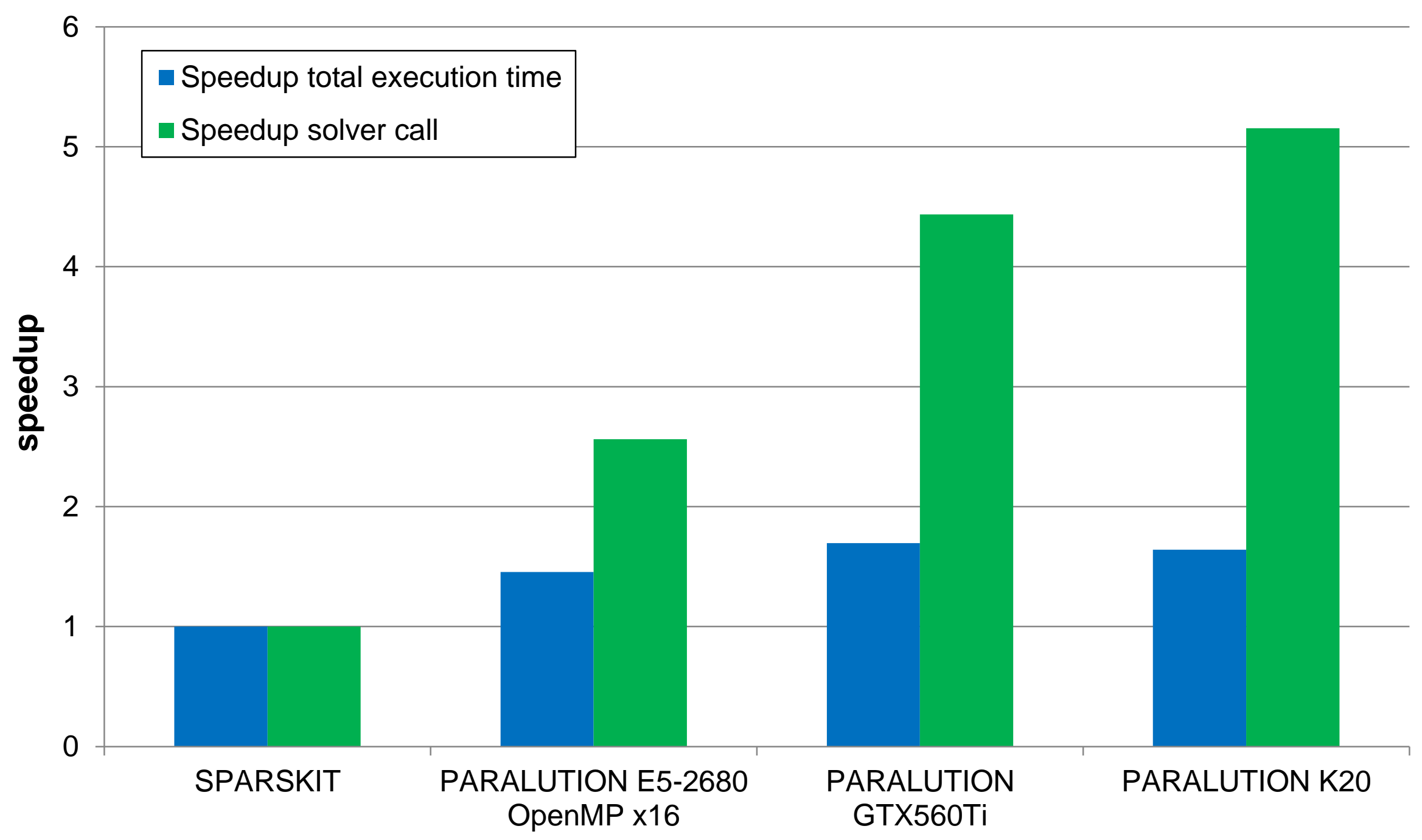




\section{COBAYA3 - some profiling results}

- Profiling results for COBAYA3 with ANDES solver and MOX case for a full simulation run:

- Sequential program parts

$63.2 \%$

COBAYA3 sequential

$63.2 \%$

PARALUTION sequential

\section{PARALUTION GPU}

COBAYA3 sequential

PARALUTION

speedup $5 x$

Speedup by Amdahl's law:

$S=\frac{1}{(1-P)+\frac{P}{N}} \leq \frac{1}{1-P}(N \rightarrow \infty)=\mathbf{1 . 5 8 2}(P=1-0.632)$ 


\section{Performance results - MOX-3D-HFP.D OpenMP scaling}

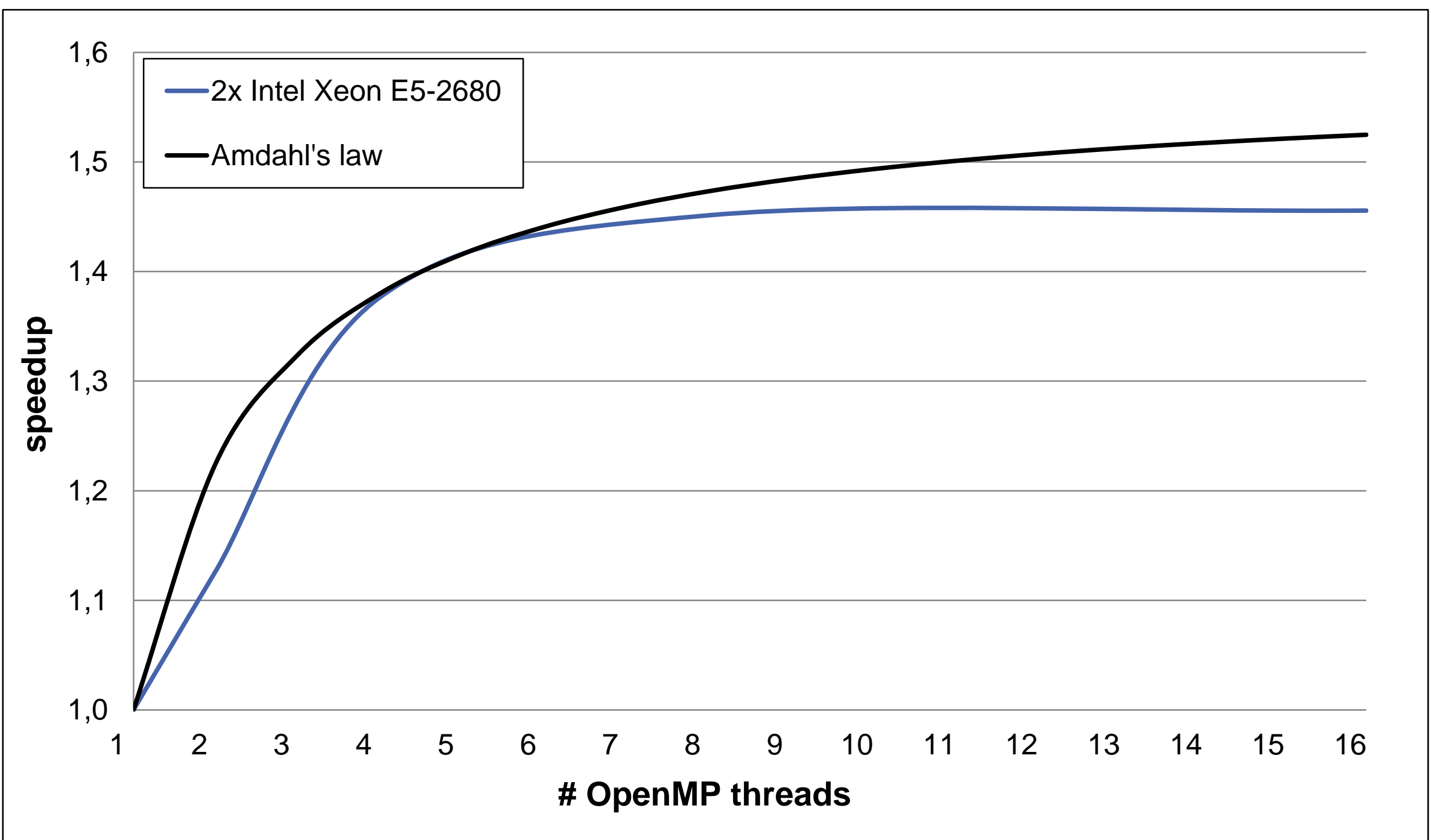




\section{Conclusion}

- PARALUTION is a very modular library, well structured

- Easy integration

- Variety of preconditioners and solvers that can easily be exchanged with the current implementation

- High portability

- Possibility to move to GPUs or upcoming new architectures without modifying existing code, e.g. Intel Xeon Phi (MIC)

- Almost no time spent by Fortran $\leftarrow \rightarrow \mathrm{C}++$ interaction

- Open source software 


\section{Outlook and future work}

- ILU-T preconditioner GPU implementation

- Not trivial to achieve fine-grained parallelism on accelerator devices, sparsity pattern is not known a-priori

- Approximate Inverse preconditioners

- SPAI, FSAI

- Coarse level parallelization using MPI

- Block Jacobi preconditioners (ILUT, ...)

- Suitability of Algebraic Multigrid

- Linear complexity!

- Suitability of PARALUTION for COBAYA3 pin by pin solver

- OpenMP implementation of the remaining sequential COBAYA3 program parts $(63.2 \%)$ 


\section{THANKS FOR YOUR ATTENTION}

